Most agree that conservative management of Thorotrast granulomas is appropriate. ${ }^{8}$ Poor healing at the biopsy site is often noted, and although healing was slow in our patient, satisfactory results were eventually obtained.

Radical neck surgery is advocated by some in an attempt to eradicate all local Thorium deposits, this has the disadvantage of a high incidence of fistula formation and does not reduce the risk of reticuloendothelial malignancy. ${ }^{8}$

Our patient seems to have had adequate treatment, and two years after this most recent presentation remained both locally free of tumour, and with no evidence of malignancy elsewhere.

Mr B Wagner and Mr M Holroyd are thanked for their assistance with EDAX analysis and the quantification of ${ }^{232} \mathrm{Th}$ exposure, as are clinical colleagues for their permission to report exposure,
This work was supported by the Yorkshire Cancer Research Campaign.

1 Gause HM, Gu Lzow M, Meyer-Hofmann G. Spatchaden nach Thorotrastawendung. L Kin Woschenschor 1966;44:32.

2 Selbie FR. Tumours in rats and mice following the injection of Thorotrast. Br J Exp Pathol 1938;19:100-7.

3 MacMahon HE, Murphy AS, Bates MI. Sarcoma of the liver: Disadvantages of Thorotrast as a diagnostic agent. Rev Gastroenterol 1947;14:155-9.

4 Abbatt JD. History of the use and toxicity of Thorotrast. Environmental Res 1979;18:6-12.

5 DiMarcangelo MT, David ET, Kuroda K. Malignant fibrous histiocytoma induced by Thorium. $N$ Engl J Med 1990;87:47-9.

6 Backer OG, Faber M, Rasmussen H. Local sequalae to carotid angiography with colloid thorium dioxide. Acta Chir Scand 1958;115:417-21.

7 Wustrow TPU, Behbehani AA, Wiebecke B. Thorotrast induced oro- and hypopharyngeal fibrosis with recurrent bleeding. J Cranio-Maxomill Fac Surg 1988;16:315-19.

8 Plent S, Shah S, Westmore GA. Thorotrast granuloma: a renascence. J Laryngol Otol 1990;104:355-7.

\title{
Synchronous squamous and glandular neoplasia of the anal canal
}

\author{
M L Yeong, K P Wood, B Scott, K Yun
}

Department of

Pathology, Wellington

School of Medicine

$M L$ Yeong

Department of

Pathology, Wellington

Hospital

K P Wood

Department of

Surgery, Wellington

Hospital

B Scott

\section{Department of}

Pathology, University of Otago, Dunedin

$\mathrm{K}$ Yun

Correspondence to:

Dr M L Yeong, Department

of Pathology, Wellington

of Pathology, Wellington

7343, Wellington South,

7343, Welling

Accepted for publication

31 July 1991

\begin{abstract}
A 48 year old man presented with invasive adenocarcinoma in the wall of a nonhealing anal fistula. The subsequent abdomino-perineal resection specimen showed residual invasive carcinoma coexisting with in situ carcinoma of anal glands as well as in situ squamous carcinoma of the anal canal. The epithelium of the anal canal had koilocytotic features. DNA hybridisation studies by the dot blot technique showed weak positivity for human papillomavirus (HPV) subtypes $16,18$.
\end{abstract}

This case illustrates a number of important points-namely, anal fistulas, particularly non-healing fistulas should be biopsied to exclude malignancy; some adenocarcinomas of the anal arise in anal glands; the coexistence of glandular and squamous carcinoma with evidence of HPV infection is highly reminiscent of similar synchronous lesions of the uterine cervix and suggests that HPV may have an aetiological role in both squamous and glandular carcinomas of the anal canal.

The anal canal is regarded as extending from the upper border of the internal sphincter to the lower border of the external sphincter and is lined by three types of epithelium. The upper third is lined by colorectal mucosa while squamous epithelium lines the distal third and is continuous with anal skin. The middle zone may be lined by squamous, columnar, or transitional epithelium which is stratified with columnar or polygonal cells superficially. Malignant tumours of the anal canal are rare. Among these squamous cell carcinoma is the most common, some of these arising in the epithelium of the transitional zone, although some may originate in the distal anus. ${ }^{1}$ There is an increasing incidence of squamous carcinoma of the anal canal, particularly among young men, with a known association of this carcinoma with human papillomavirus (HPV) infection. ${ }^{2}$ Rarer still is the adenocarcinoma of the anal canal, the origin of which is often debated.

This is a report of a patient with, uniquely, an invasive adenocarcinoma occurring at the same time as in situ glandular and squamous cell carcinoma of the anal canal, which may shed light on the origin and pathogenesis of these tumours.

\section{Case report}

A 48 year old previously well, married man presented with an anal fistula which failed to heal despite initial surgery. With further surgery and a wedge biopsy of the wall of the fistula, an invasive adenocarcinoma was found. The patient then had an abdominoperineal resection from which he made an uneventful recovery. 
Figure 1 Two anal glands showing changes of carcinoma in situ. There is an abnormal complex branching and budding pattern and lining cells (inset) show crowding, loss of polarity, and

pronounced nuclear atypia (haematoxylin and eosin).

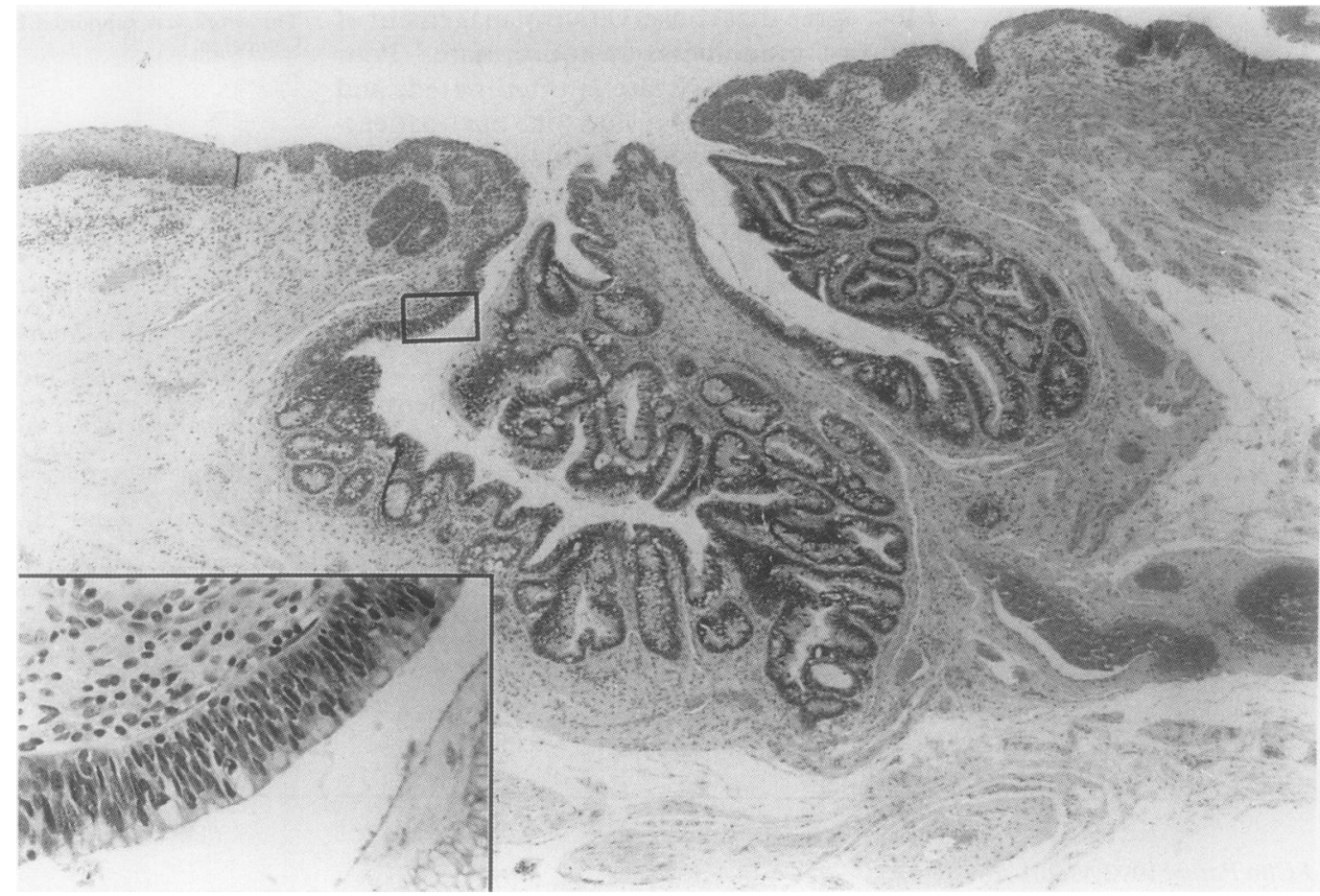

All tissues were fixed in $10 \%$ formol-saline and stored in paraffin wax. Histological sections were stained with routine haematoxylin and eosin. For dot blot hybridisation, ${ }^{3} 10 \mu \mathrm{m}$ sections were dewaxed, treated with proteinase $\mathrm{K}$ digestion buffer, and incubated for two days. The digest was spun at $10000 \times g$ and the supernatant directly dotted on nitrocellulose. After denaturation and prehybridisation the specimen was hybridised with biotinylated HPV probes 16,18 , and 31,33 . After washing with high and low stringency solutions detection was achieved by binding with streptavidin alkaline phosphatase reacting with 5 bromo 4 chloro 3 indoyl phosphate-nitro blue tetrazolium substrate.

\section{Pathology}

The deep tissues of the wedge biopsy specimen were infiltrated by a tumour composed of small tubules as well as larger glands, some with complex papillary projections. There was surrounding desmoplasia and infiltration by lymphocytes. The abdomino-perineal resection specimen showed residual invasive carcinoma in the anal canal. In the adjacent tissue some normal anal glands lined by anal transitional epithelium were identified. Other glands, however, were lined by highly atypical crowded columnar cells with pronounced nuclear atypia, some containing cytoplasmic mucin. These glands showed budding and seemed to open normally into the anal canal which, at this point, was covered by transitional mucosa (fig 1). The adjacent squamous epithelium showed the full thickness changes of carcinoma in situ. Some superficial cells of this epithelium showed densely stained nuclei with perinuclear haloes reminiscent of HPV associated lesions of the uterine cervix (fig 2). Dot blot hybridisation studies showed a weakly positive reading for HPV subtypes $16,18$.
Figure 2 Squamous carcinoma in situ of the anal canal, including an epithelial whor (thick arrow). Superficially, koilocytes with dense "India ink" nuclei, perinuclear haloes, and eosinophilic cytoplasm are present (thin arrows), (haematoxylin and eosin). 


\section{Discussion}

Adenocarcinoma of the anal canal is rare. The tumour may present as an obvious mass lesion in the anal canal but may lie undetected in a perirectal abscess or a chronic anal fistula. ${ }^{45}$ Biopsy of all perianal abscesses and fistulas is therefore recommended. The origin of adenocarcinoma of the anal canal is often debated but its common association with a perianal abscess or anal fistula prompted the suggestion that anal adenocarcinomas arise in a pre-existing anal fistula. Other theories suggest that it may originate in reduplication of the gut, implantation from a rectal carcinoma, or in anal glands of the anal transitional zone. ${ }^{45}$ The association of this patient's adenocarcinoma with an in situ lesion of the anal glands is strong evidence that some adenocarcinomas of the anal canal arise from anal glands. The association of anal gland carcinoma with in situ squamous carcinoma of the anal canal has not to our knowledge been reported previously and is reminiscent of similar lesions in the uterine cervix. ${ }^{6}$ The association of HPV with squamous carcinomas of the anal canal has been established in recent years. Koilocytes were identified in this patient's tissues, although dot blot hybridisation showed only weak positivity for HPV subtypes 16,18 . This may be due to the presence of low viral copies and the relatively low sensitivity of dot blot hybridisation. It is tempting to postulate that, as in the uterine cervix, HPV infection may have an aetiological role in both glandular and squamous carcinomas of the anal canal.

\section{Conclusion}

The presentation of the invasive lesion as a non-healing anal fistula reinforces the need to biopsy these lesions to exclude malignancy. The identification of an in situ carcinoma of anal glands lends support to one theory that anal adenocarcinoma may arise from anal glands. Most interestingly, simultaneous glandular and squamous carcinoma associated with HPV infection suggests an analogy with similar simultaneous lesions in the uterine cervix and may point to an aetiological role for HPV in primary adenocarcinoma of the anal canal.

1 Fenger C. Anal canal tumours and their precursors. Pathology Annual Part 1. East Norwalk, Connecticut: Appleton \& Lange, 1988:45-66.

2 Talbot $R$. Changing nature of anal cancer. $B M J$ 1988;297:239-40.

3 Schneider A, Grubert T. Diagnosis of human papilloma virus infection by recombinant DNA techniques. Clin Obstet Gynaecol 1989;32:127-40.

4 Zaren HA, Delone FX, Lerner HJ. Carcinoma of the anal gland; case report and review of the literature. $J$ Surg Oncol 1983;23:250-4.

5 Nelson RL, Prasad ML, Abcarian H. Anal carcinoma presenting as a perirectal abscess or fistula. Arch Surg 1985;120:632-5.

6 Anderson MC. Premalignant and malignant disease of the cervix. Obstetrical and gynaecological pathology Vol I 3rd ed. Edinburgh: Churchill Livingstone, 1987:289.

\title{
Combined high grade sarcoma and serous ovarian neoplasm
}

\author{
C Allen, $M$ Stephens, J Williams
}

\author{
Histopathology \\ Department, Central \\ Pathology Laboratory, \\ Hartshill, Stoke on \\ Trent ST4 7PA \\ C Allen \\ M Stephens \\ Department of \\ Radiotherapy, North \\ Staffordshire Royal \\ Stafiordshire Royal
Infirmary, Hartshil, \\ Stoke on Trent \\ J Williams \\ Correspondence to: \\ Dr M Stephens \\ Accepted for publication \\ 27 August 1991
}

\begin{abstract}
A case of an ovarian serous epithelial neoplasm of borderline type admixed with sarcomatous elements is reported. This combination seems to be extremely rare with only four cases previously reported. It may represent a type of collision tumour or the development of a sarcoma in a growth with borderline differentiation.
\end{abstract}

\section{Case report}

CLINICAL FEATURES

A 63 year old woman (gravida 2 para 2 ) with no relevant medical history presented with a two week history of lower abdominal pain and distension. Examination showed a tender, firm mass arising from the pelvis and extending to the left of the umbilicus. At laparotomy she was found to have ascites, a left ovarian tumour, equivalent in size to a 20 week gestation, probable tumour in the smaller right ovary, and probable deposits within the pouch of Douglas, omentum, and para-aortic nodes. Most of the left ovary was removed with difficulty. The right ovary was adherent to bowel and could not be dissected free. Lymph nodes, omentum, and peritoneum were not sampled. The patient was subsequently given chemotherapy. She died 11 months after diagnosis. 